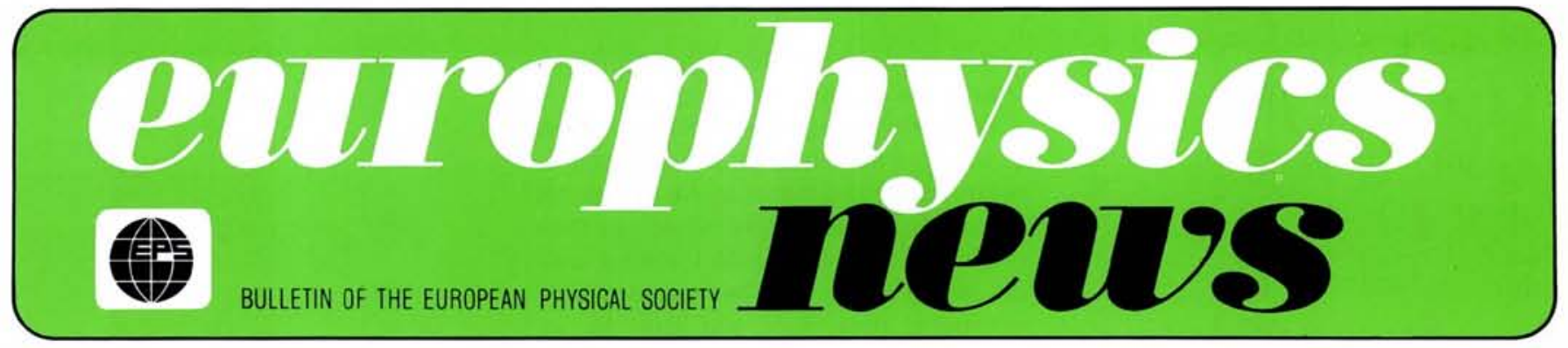

J.A. Volume 18

\title{
Rare Earth-Transition Metal Permanent Magnets
}

\section{Givord, Grenoble}

\author{
(Laboratoire Louis Néel, C.N.R.S.)
}

I-From AINiCo's to Neodymium-IronBoron Permanent Magnets

In several instances, since the beginning of this century, the discovery of new magnetic materials has strongly influenced the development of permanent magnets. The first very important advance was made in 1932 in Japan. Mishima prepared magnets by thermal treatment of an alloy based on iron, nickel and aluminium, that led to a new range of alloys now known as AINiCo. The energy product attained was an order of magnitude larger than that of any previously known magnetic material. In 1953, low cost ferrites were discovered in the Philips laboratories. These have the remarkable property of being strongly resistant to induction fields which tend to reverse the magnetic moment, i.e. they have a high coercivity.

In those two families of magnets, the magnetic properties are intrinsically limited by the fact that the magnetism of the transition elements iron, cobalt and nickel only is involved. In 1965, Hoffer and Strnat ") pointed out that the rare earth transition metal (R-M) alloys have specific magnetic properties which made them very promising for permanent magnets and in the years following, permanent magnets were prepared based on the $\mathrm{SmCo}_{5}$ compound. The magnetic properties of this family of magnets were improved over the next 20 years to such an extent that these materials could compete with electromagnets in many applications. However, cobalt is the most expensive of the magnetic transition elements, and samarium the most expensive of the light rareearth elements: the price of $\mathrm{SmCo}_{5}$ alloys is to-day about $100 \$ / \mathrm{kg}$. For this reason, the $\mathrm{Sm}$-Co magnets have been used for very specific applications only.

Because $\mathrm{Fe}$ is much less expensive than $\mathrm{Co}$, it is a priori desirable to prepare magnets based on R-Fe alloys, but no stable binary compound in this system exhibits all the properties required. Consequently in the 1970s, several groups, in particular in the United States, were very actively looking for new appropriate metastable magnetic phases which could be stabilized by very rapid quenching of alloys from the melt, or by thermal annealing of amorphous alloys.

At the conference on Magnetism and Magnetic Materials held in Pittsburgh in November 1983, Sagawa et al. ${ }^{2}$ ) from the Japanese company Sumitomo reported on the preparation of magnets using a traditional powder metallurgy method but starting from an alloy of chemical composition $\mathrm{Nd}_{15} \mathrm{Fe}_{77} \mathrm{~B}_{8}$. Their results revealed that a new phase exhibiting the properties required for permanent magnet materials was formed between rare-earths, such as neodymium, and iron when a relatively modest amount of boron was included. It is interesting to note that very likely the boron was initially added accidentally to the alloy as a result of a $\mathrm{Nd}-\mathrm{Fe}$ alloy being melted in a boron nitride crucible. At the same Pittsburgh conference, other groups ${ }^{3-5}$ ) in particular from General Motors, reported similar results, although not quite as good, starting from alloys obtained by rapid quenching. Following a route entirely different to that of Sagawa et al., these groups had included boron in their alloys in order to make amorphicity easier. Here again, they found accidentally the new ternary $\mathrm{R}-\mathrm{Fe}-\mathrm{B}$ phase.

Using this newly discovered magnetic phase, permanent magnets with unparalleled properties can be prepared. In addition, the price of the basic Nd-Fe-B alloy is nearly an order of magnitude cheaper than that of the Sm-Co alloys. Applications in many different areas will certainly develop in the near future, ranging from sensors, loudspeakers, motors to undulators, NMR imaging magnets and even Maglev (magnetic levitation) transportation systems. As a consequence, several groups all over the world have become interested in this new material. In particular, a "Concerted European Action on Magnets" has been established by the European Commission to encourage collaborations at a European level ${ }^{6}$ ); more than 50 laboratories are involved. Further progress is possible in several different directions. i) It must be remembered that the $\mathrm{Nd}$ Fe-B phase was essentially found by

\section{Contents}

Rare Earth-Transition
Metal Permanent
Magnets

Cooling Neutron Stars

European Suppression Shield Array

93

98

New Members of EPS

First EPS ACAPPI Workshop

102

102

European Geotraverse Project

103

104 


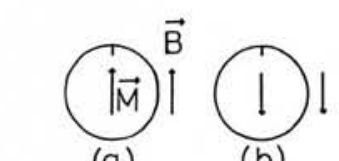

(a)

(b)<smiles>C1=CCCCCCCC1</smiles>

(c)

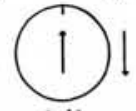

(d)

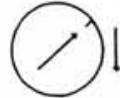

(e)

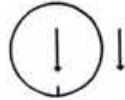

( $f$ )
Fig. 1 - Behaviour of a magnetic moment $\vec{M}$ under the influence of an induction field $\vec{B}$. (a) and (b): ordinary ferromagnet. The moments rotate in order to remain parallel to the field.

(c) to (f): permanent magnet. The moments cannot rotate freely inside the material. (d) As the field is reversed they become antiparallel to $\vec{B}$. In (e) and (f) the whole specimen rotates in order to minimize the energy.

chance, so it is possible that other suitable phases could be prepared.

ii) Recent economic assessements suggest that the development of these new magnets will be limited mainly by the relatively high cost of fabrication. Original and cheaper methods of fabrication should thus be sought.

iii) Permanent magnets, in general, are used as parts of more complex devices. These, in many instances, should be redesigned in order to take full benefit of the improved magnetic properties.

\section{II - Properties required:}

Ferromagnetism and Coercivity

In ferromagnetic materials, the atomic magnetic moments $\mu_{\text {at }}$ created by the motion of the electrons are parallel, the total moment being $M=N \mu_{\text {at }}$, where $N$ is the number of atoms. At the origin of this parallel coupling of the magnetic moments are exchange interactions. Let us consider an ordinary ferromagnet under the influence of an induction field $\vec{B}$ where the magnetostatic energy is

$$
E_{\mathrm{M}}=-\vec{M} \vec{B}
$$

The magnetic moments tend to align along the induction field so that as the direction of the field is reversed, they rotate inside the material in order to remain parallel to the field (Fig. 1).

Permanent magnets are ferromagnets in which the moments are not free to rotate inside the material. As the field is reversed, the moments remain in their original direction and so become antiparallel to $B$ (Fig. 1). The magnetostatic energy is maximum and can, for instance, be transformed into motion if the whole specimen is allowed to rotate. The property of coercivity, whereby the magnetic moments do not rotate easily inside the material is linked to an interaction between the moments and the crystalline lattice. As a result, the energy of the magnetic moments depends on their direction and the phenomenon is anisotropic.

\section{III - Ferromagnetism and Magnetic Anisotropy \\ Origin of ferromagnetism}

The exchange interactions at the origin of ferromagnetism arise from the electrostatic repulsion between electrons. Electrons tend to avoid each other and thus do not sit on the same orbital. Moreover, associated with each electron is an intrinsic magnetic moment, called the spin magnetic moment, and the electrostatic repulsion is further decreased when the spin moments of electrons on different orbitals are parallel. The probability then for one electron to hop from one orbital to the other is strictly zero since, according to the Pauli exclusion principle, two electrons of the same spin state cannot occupy the same orbital.

The exchange interactions are strongest for iron, cobalt and nickel. In these metals, the magnetic electrons occupy the outer $3 d$ shell and form part of the conduction band, i.e. the orbitals extend over several atomic sites. Thus, the interactions between electrons on different sites are strong, and lead to parallel

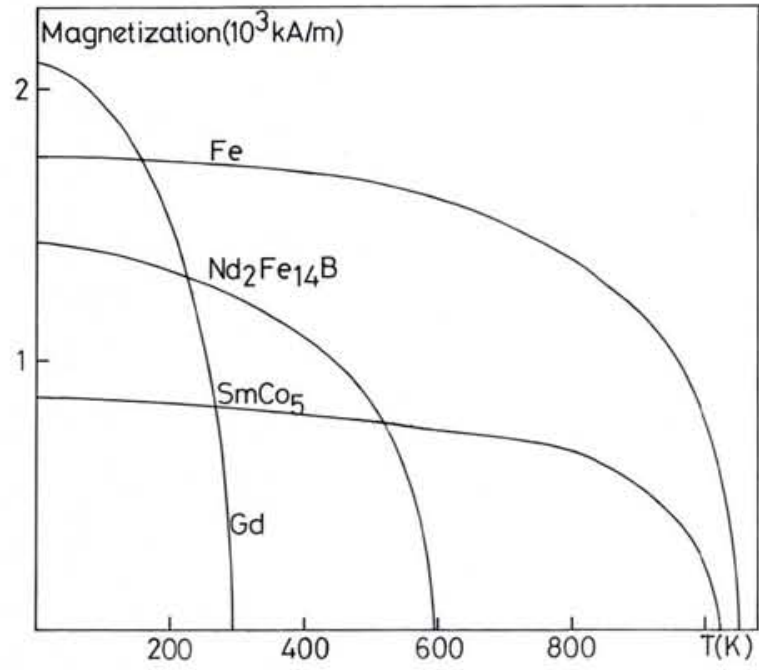

Fig. 2 - Temperature dependence of the magnetization and Curie temperature in iron, gadolinium, $\mathrm{SmCO}_{5}$ and $\mathrm{Nd}_{2} \mathrm{Fe}_{14} \mathrm{~B}$.

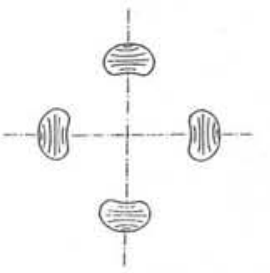

(a)

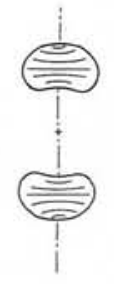

(b)

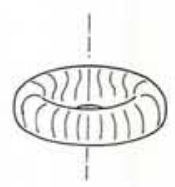

(c)
Fig. $3-3 d$ and $4 f$ orbitals. (a) the symmetry of the $3 d$ orbitals is that of the surroundings, giving rise to weak orbital moment. The symmetry of the $4 f$ orbitals is such that the orbital moment is large. The $4 f$ electron density may be prolate (b) or oblate (c).

coupling of the spin magnetic moments throughout the whole metal.

The magnetization $J=M N$ can be defined as the magnetic moment per unit volume; it reaches $1740 \mathrm{kA} / \mathrm{m}$ in iron at $4.2 \mathrm{~K}$. As the temperature increases, the magnetization decreases owing to the effect of thermal disorder on the coupling between the magnetic moments, vanishing at the Curie temperature, $T_{c}$. In iron, $T_{c}=1041 \mathrm{~K}$ (Fig. 2). In contrast, in the rare-earth elements, the electrons responsible for magnetism occupy the inner $4 \mathrm{f}$ shell and do not form part of the conduction band. As a result, the electrostatic interactions, and in particular the exchange interactions between electrons on different sites are weak. Ferromagnetism in these metals is destroyed by thermal activation at temperatures below room temperature, as shown in Fig. 2 for gadolinium.

\section{Magnetic anisotropy}

Magnetic anisotropy is due to a local coupling of the moments at each atomic site with the crystalline lattice. The anisotropy energy is minimum when the moments point along the easy magnetization direction and a maximum when they point along the difficult direction. A measure of magnetic anisotropy is given by the value of the anisotropy field, $B_{\text {ani' }}$ which is the field required to rotate the moments from their easy direction to their difficult direction. Magnetic anisotropy does not involve directly the spin magnetic moments. Rather, it occurs when a specific contribution to the atomic moment called the orbital moment is present. This orbital moment, is the direct analog on an atomic scale, of the magnetic moment of an electrical circuit on a macroscopic scale. The more the orbitals differ from spherical symmetry and tend to uniaxial symmetry, the larger the orbital moment.

Because of the outer character of the $3 \mathrm{~d}$ shell in transition metals, the $3 \mathrm{~d}$ orbitals are strongly influenced by the electrostatic charges of neighbouring 
ions. Their symmetry is that of the surroudings with, in general, several symmetry axes (Fig. 3). The resulting orbital moment is small leading to weak magnetic anisotropy. In cobalt, $B_{\text {ani }}$ is about $1 \mathrm{~T}$.

In rare earth metals, a strong interaction occurs between spin and orbital moments which favours electron orbitals whose orbital moment is maximum. In metals such as samarium, the $4 \mathrm{f}$ electron density is prolate, i.e. elongated along the unique axis like a cigar; in metals such as neodymium, the $4 \mathrm{f}$ electron density is oblate, i.e. flat like a pancake (Fig. 3 ).

The magnetic anisotropy then results from the interaction of the $4 \mathrm{f}$ negative electron density with the charges of the ions in the environment. It is large when the environment favours a unique easy magnetization axis. Let us consider, for example, that the surrouding charges are positive and in a given plane. The $4 f$ electron density, attracted by the positive charges of the ions, is thus confined to this plane. For oblate electron density, the moments are perpendicular to the plane whereas for prolate electron density, two situations of equivalent energy occur, with the moments in the plane (Fig. 4). In rare-earth metals, the anisotropy fields at low temperatures may be as large as $50 \mathrm{~T}$.

IV - Combination of Ferromagnetism and Large Magnetic Anisotropy in Rare Earth-Transition Metal-Rich Alloys

It was shown in the previous section that ferromagnetism above room temperature is a characteristic of transition metal magnetism, whereas large magnetic anisotropy is observed in rareearth metals. Both these properties are required in permanent magnet materials and their optimum combination is observed in rare earth-transition metal-rich alloys.

In particular, in $\mathrm{RCo}_{5}$ compounds, the exchange interactions between $3 \mathrm{~d}$ electrons stabilize ferromagnetism up to $900 \mathrm{~K}$ (Fig. 2). Magnetic anisotropy is due to the rare-earth $4 \mathrm{f}$ electrons and is large if the easy magnetization direction is the unique axis ( $\vec{c}$-axis) of the hexagonal structure (Fig. 5). The ionic charges which have the largest effect on a given $R$ atom are those of the rare-earth ions in the adjacent planes. The $\vec{c}$-axis is thus that of easy magnetization in the case of a prolate $4 \mathrm{f}$ electron density, like in Sm. In the compound $\mathrm{SmCo}_{5}$, the anisotropy field $B_{\text {ani }}$ reaches $35 \mathrm{~T}$ at room temperature.

None of the binary R-Fe compounds exhibit large exchange interactions and
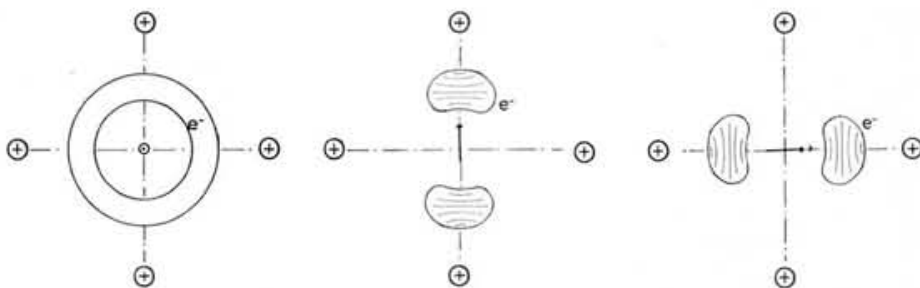

Fig. 4 - Magnetic anisotropy. The orientation of the 4 f electron density is determined by the interaction with the surrounding charges. In this example, the moment direction is perpendicular to the plane for an oblate electron density and two in-plane equivalent directions occur for a prolate electron density. uniaxial anisotropy. In particular, it is an experimental fact, the description of which is beyond the scope of this article, that exchange interactions between $\mathrm{Fe}$ moments are strongly reduced when very short $\mathrm{Fe}-\mathrm{Fe}$ interatomic distances are present. In the ternary $\mathrm{R}_{2} \mathrm{Fe}_{14} \mathrm{~B}$ compounds, the interatomic distances are not as short as in R-Fe-rich binary compounds. The Curie temperatures are of the order of $600 \mathrm{~K}$ (Fig. 2).

Although not as large as in the $\mathrm{RCo}_{5}$ compounds, they are high enough for room temperature applications to be possible. The tetragonal crystal structure of these compounds is shown in Fig. 5. Here, the nearest neighbours of a given $R$ atom lie in an atomic plane, perpendicular to the $\vec{c}$-axis. The $4 \mathrm{f}$ electron density is confined to this plane, thus leading to uniaxial anisotropy for rare-earth elements where the $4 \mathrm{f}$-electron density is oblate, i.e. praseodymium (Pr) or neodymium (Nd). In $\mathrm{Nd}_{2} \mathrm{Fe}_{14} \mathrm{~B}$, the anisotropy field at room temperature, $B_{\text {ani }}$, is about $10 \mathrm{~T}$.

\section{V - Theoretical Versus Experimental Coercive Field}

It was shown in section II that, in a permanent magnet, the magnetization can be along a direction opposite to that of the applied magnetic field $B_{\text {app }}$. However, as the field strength is increased, the magnetization reverses at a critical value of the applied field, called the coercive field, $B_{c}$. The reversal process is schematized in Fig. 6-a. At the initial and final stages, the moments lie along the easy axis. They are along the difficult axis at an intermediate stage which corresponds to an energy barrier:

$$
\Delta E_{\mathrm{M}}^{\text {easy-diff. }}=E_{\text {ani }} \times V
$$
where $E_{\text {ani }}=1 / 2 \mathrm{~J} \times B_{\text {ani }}$ is the anisotropy energy per unit volume lost when the moments are along the difficult axis and $V$ is the volume of the sample. $E_{\text {ani }}$ in rare earth-transition metal compounds is about $10^{8} \mathrm{~J} / \mathrm{m}^{3}$. It can thus be shown that the theoretical value of the coercive field, $B_{c}$, required to overcome the barrier, is equal to $B_{\text {ani }}$.

In typical ferromagnetic materials, the experimental value of the coercive field is several orders of magnitude weaker than the theoretical value. It appears that magnetization reversal is not an inphase rotation of all the moments as schematized above, rather does it occur according to the scheme of Fig. 6-b. The intermediate state during magnetization reversal is such that magnetization is

\section{UNIVERSITY OF LIVERPOOL Chadwick Fellowships}

\section{Department of Physics}

In honour of Sir James Chadwick, Nobel Laureate and Professor of Physics, (19351948), the University intends to support new positions for young experimental physicists of outstanding merit who are $\mathbf{3 0}$ years or under at the date of application. The holders of these Fellowships are expected to make contributions to both the research and teaching programmes of the Department.

The present research activities are concentrated in the areas of Nuclear, Elementary Particle and Solid State Physics, exploiting both the N.S.F. and S.R.S. facilities at the nearby Daresbury Laboratory and the high energy facilities at CERN (Geneva) and DESY (Hamburg) as well as excellent departmental facilities.

Appointments will be for an initial period of three years and can be extended to five years. Information on the research and teaching programmes can be obtained from the Head of the Department, Professor E. Gabathuler, Tel. (51) 70960 22, Ext. 2275.

The salary will be within the range $f 8,185-£ 12,605$ per annum.

Applications, consisting of curriculum vitae, a list of publications, together with the names of three referees, should be received not later than 1st September, 1987, by The Registrar, The University, P. O. Box 147. Liverpool L69 3BX, UK

from whom further particulars may be obtained.

Quote ref. RV/531/EN 
Fig. 5 - Hexagonal structure of the $\mathrm{RCO}_{5}$ compounds and tetragonal structure of the $\mathrm{R}_{2} \mathrm{Fe}_{14} \mathrm{~B}$ compounds.

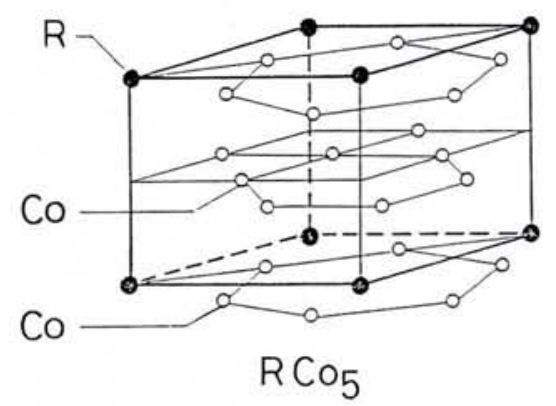

reversed in a part of the specimen, not in the other. These so-called "magnetic domains" are separated by a domain wall in which the atomic magnetic moments progressively rotate from one direction to the other (Fig. 6-b). The magnetic energy lost in domain wall creation is

$$
\Delta E_{M}^{\text {domain }}=E_{\mathrm{W}} \times S
$$

where $E_{W}$ is the magnetic energy per unit area lost in the wall and $S$ the area of the wall. A first contribution to $E_{\mathrm{w}}$ arises from the magnetic moments in the wall not being parallel as required by exchange interactions. However, the rotation of the moments is progressive in such a way that neighbouring atoms are almost parallel. As the range of exchange interactions is very short, the energy loss is reduced. The second contribution to $E_{\mathrm{W}}$ arises from anisotropy energy, the moments not being parallel to their easy direction. In rare earthtransition metal compounds, $E_{\mathrm{W}}=0.1$ $\mathrm{J} / \mathrm{m}^{2} . \Delta E_{M}^{\text {easy-diff. }}$ and $\triangle E_{\mathrm{M}}^{\text {domain }}$ are of the same order of magnitude for a sample volume of typically $(50 \AA)^{3}$. As the volume of the sample increases, $\triangle E_{M}^{\text {domain, }}$, proportional to the surface of the walls, increases much slower than $\Delta E_{\mathrm{M}}^{\text {easy-diff. }}$. The latter is several orders of magnitude larger than the former in samples of macroscopic size.

The above arguments suggest that magnetization reversal through domain wall creation is highly favoured. However, in a "perfect" material, there is no

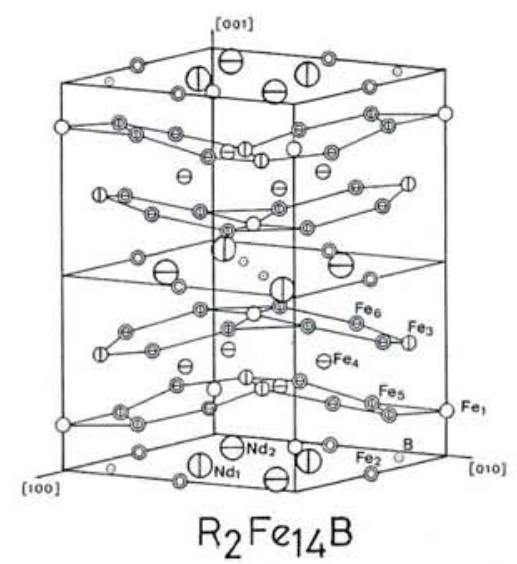

possible way of creating such a wall and the value of the coercive field should not be reduced. Crystal defects are essential in this context, as they render the creation of a domain wall much easier at some point in the crystal and are consequently at the origin of the drastic reduction observed of the coercive field. The larger the difference between $\triangle E_{M}^{\text {easy-diff. }}$ and $\triangle E_{M}^{\text {domain }}$, the stronger is the influence of crystal defects and consequently the larger is the reduction of coercivity.

\section{VI - Permanent Magnets Fabrication}

For the coercivity to increase, it is necessary to reduce the influence of crystal defects. This is the art of permanent magnet fabrication. The difference between $E_{M}^{\text {domain }}$ and $E_{M}^{\text {easy-diff. is reduced as }}$ the volume of the sample is reduced and this suggests the use of a fine-powder magnetic material. The technology of $\mathrm{SmCo}_{5}$ permanent magnet preparation was established in the 1970 s by Benz and Martin ${ }^{7}$ ) and Das et al. ${ }^{8}$ ). Similar technology has recently been employed for the Nd-Fe-B by Sagawa et al. 1). The ferromagnetic material is first ground into a fine powder and then compressed and sintered to almost full density. In the bulk specimen obtained, the defects at the grain boundaries are sufficient to cancel the very short-ranged exchange interactions between grains and the sample behaves like an assembly of independent grains of typical size $10 \mu \mathrm{m}$.

Fig. 6 - Magnetization reversal processes. (a) in-phase rotation of all the moments. In this case the theoretical value of the coercive field is $B_{C}=B_{A}$, the anisotropy field. (b) reversal of magnetization by creation and growing of magnetic domains. This process, strongly influenced by structural defects, leads to a strong reduction of the coercive field, $B_{C}<<B_{A}$. The art of permanent magnet fabrication is to find metallurgical processes which reduce the influence of defects and thus increase $B_{C}$.
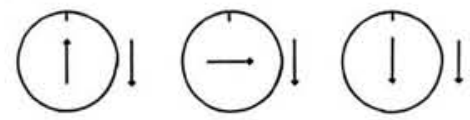

(b)
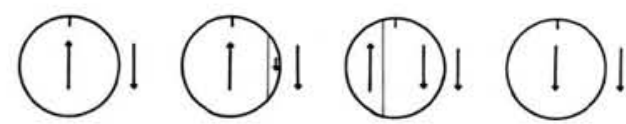

Coercive fields $B_{c}$ of the order of $1 / 10$ $B_{\text {ani }}$ are reached. This is still much less than the theoretical value, but the value obtained (1.2 T) is large enough for most practical applications.

Another mechanism may be responsible for the establishment of coercivity. In an inhomogeneous material, the value of $E_{\mathrm{W}}$ is not the same throughout and the magnetic domains tend to be pinned at positions of minimum energy. Profit from this idea was first taken by Nesbitt et al. ${ }^{9}$ ) in the $\mathrm{Sm}(\mathrm{Co}-\mathrm{Cu})_{5}$ system. The magnetic domains are pinned in $\mathrm{Cu}$-rich regions where, $\mathrm{Cu}$ atoms being nonmagnetic, the exchange interactions are reduced. Thus, as the induction field is increased, magnetic domains of reversed orientation are created, but they do not grow. Neglecting the small volume of the domains of reversed magnetization, it may be said that the moment of the magnet is opposed to the field. At the coercive field, the magnetic walls become unpinned. In these systems, $B_{c}$ is again of about $0.1 B_{\text {ani. In Nd-Fe-B ma- }}$ gnets, several authors ${ }^{3-5}$ ) have shown that magnetic inhomogeneities can be developed by the fast quenching of alloys from the melt leading to coercivity of similar origin.

\section{Conclusion}

In conclusion, the rare earth-transition metal alloys present a unique association of high temperature ferromagnetism and large magnetic anisotropy. This has led to a considerable improvement in permanent magnet properties. The recent discovery of the $\mathrm{Nd}_{2} \mathrm{Fe}_{14} \mathrm{~B}$ family makes possible the development of low cost Fe-based magnets. However, it must be noted that the cost of fabrication is still rather high.

\section{REFERENCES}

1. Hoffer G. and Strnat K., I.E.E.E. Trans. Mag. 2 (1966) 487.

2. Sagawa M., Fujimura S., Togawa M., Yamamoto $\mathrm{H}$. and Matsuura Y., J. Appl. Phys. 55 (1984) 2083

3. Koon N.C. and Das B.N., J. Appl. Phys. 55 (1984) 2063.

4. Croat J.J., Herbst J.F., Lee R.W. and Pinkerton F.E., J. Appl. Phys. 55 (1984) 2078.

5. Hadjipanayis G.C., Hazelton R.C. and Lawless K.R., J. Appl. Phys. 55 (1984) 2073. 6. The "Concerted European Action on Magnets" can be obtained from I.V. Mitchell, Commission of the European Communities (Brussels).

7. Benz M.G. and Martin D.L., Appl. Phys. Lett. 17 (1970) 176.

8. Das D.K., I.E.E.E. Trans. Mag. MAG-5 (1969) 214.

9. Nesbitt E.A., Willens R.H., Sherwood R.C., Buehler E. and Wernick J.H., Appl. Phys. Lett. 12 (1968) 361. 